
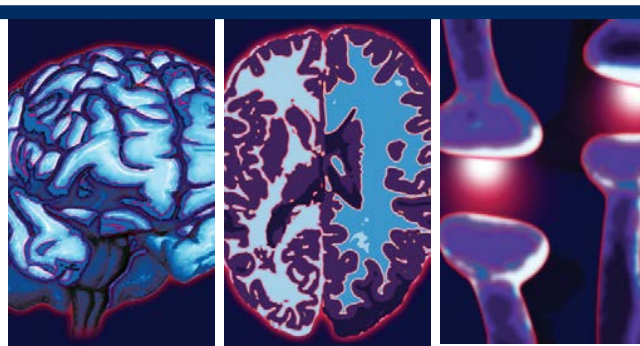

\title{
A Rare Case of Non-Convulsive Status Epileptic with Psychotic Presentation
}

\author{
Ana Samico ${ }^{\dagger}$ Ângela Venâncio
}

\begin{abstract}
\section{Background}

Psychosis associated with epilepsy is one of the temporal and frontal lobe epilepsy (TFLE) forms of presentation. Non-convulsive epileptic status (NCSE) clinical features are subtle and non-specific and, consequently, they are generally not diagnosed and confused with behavioral or psychiatric disorders.

It remains a challenge to characterize neurobiological changes that contribute to the genesis or maintenance of both conditions, with a clear need for further investigation.
\end{abstract}

\section{Case report}

A 56-year-old male patient was hospitalized due to a one month period with persecutory delusions, auditory hallucinations, thought diffusion, passivity phenomena and total insomnia. There were no relevant changes in the general and neurological physical examinations, analytical study, urine's substance abuse drugs and cerebral CT.

His psychotic symptoms diminished with paliperidone, but one month after his admission he began to present periods of confusion, consciousness floating, temporal and spatial disorientation, disperse attention, behavioral disorganization at night and ataxia.

He realized an electroencephalogram (EEG) and had practically continued epileptic activity in the temporal and frontal lobes, which ceased with the addition of valproic acid (VPA).

Four months later the patient was discharged, medicated with paliperidone and VPA, with no psychotic symptomatology.

\section{Conclusions}

Today the most accepted theory is that both Psychosis and Epilepsy are the consequence of underlying neuropath physiological dysfunction, going towards the specter of a Psychosis's "continuum".

The NCES can have a variety of clinical presentations and the EEG is necessary to make a definitive diagnosis, but this can be difficult especially due to its low availability and because there is currently no consensus on the EEG diagnostic criteria.

This clinical case was particularly challenging and the diagnostic delay could represent a liability to the patient's recovery.

In both pathologies, the treatment remains a challenge for psychiatrists and neurologists, and further studies are needed to clarify the best diagnostic and therapeutic approach. 


\section{Case Report Ana Samico}

\section{Keywords}

Temporal lobe epilepsy, Non convulsive status epileptic, Psychosis

\section{Introduction}

Psychosis associated with Epilepsy is one of the presentation forms of Temporal and Frontal Lobe Epilepsy (TFLE), a neurological condition in which seizures can be generalized and affect a whole set of limbic and neocortex structures [1].

The prevalence of psychiatric disorders is greater in people with Epilepsy than in the general population (2) and the percentage of epileptic patients who present with psychotic illness varies between $5.6 \%$ and $80 \%$ [2-4].

The lack of consistency of data is probably due to methodological differences in their study, such as changes in diagnostic classifications, clinical heterogeneity and different verification methods. This lack of rigor is also reflected in the fact that its etiology and pathophysiology have not yet been clearly established [4,5].

Although the combination of anti-epileptics and antipsychotics has been generalized as a therapeutic approach, there are no standardized treatment guidelines for Psychoses associated with Epilepsy [6].

The Non-convulsive Status Epileptic (NCSE) accounts for approximately $25 \%$ of status epilepticus and persists in $14 \%$ of patients following control of generalized epilepsy [7].

Although both conditions, TFLE and NCSE, require immediate diagnosis and intervention, the clinical features of NCSE are subtle and nonspecific and therefore are not usually diagnosed and confused with behavioral or psychiatric disorders [8]. The etiology, diagnosis, treatment, and prognosis remain controversial $[9,10]$.

The objective of this paper is to clarify the clinical and therapeutic characteristics of Psychosis associated with Epilepsy and the NCSE, with a view to a greater and better recognition and approach of these conditions, both psychiatric and neurological level, with reference to a clinical case.

\section{Case report}

A 56-year-old male patient was compulsory hospitalized due to a one month period with persecutory delusions, auditory hallucinations, thought diffusion, passivity phenomena and total insomnia. He had been self-medicated with diazepam $40 \mathrm{mg}$ per day due to the insomnia, with no response, and had no insight for the remaining clinical presentation. There were no relevant changes in the general and neurological physical examinations, analytical study (with hemogram, renal and hepatic function, ionogram, cholesterol and triglycerides, thyroid function, HIV, Hepatitis B and C and syphilis serologies, summary urine analysis), urine's substance abuse drugs and cerebral CT.

He described this one-month period as "a dream...but it does not happen during sleep, it's awake...where things that I can't control happen and my body does things that shouldn't do".

Approximately ten years before, he had what was described as a "depressive episode", but neither the patient nor his family could accurately describe it and there were no follow-up records. He had no other relevant medical history.

His psychotic symptoms improved with paliperidone $9 \mathrm{mg}$, but the insomnia remained despite the therapeutic adjustments.

One month after his admission he began to present periods of confusion, consciousness floating, temporal and spatial disorientation, disperse attention, behavioral disorganization at night and ataxia, that doesn't improve despite therapeutic. Psychiatric medication, including paliperidone was ceased and he had no changes in the neurologic status, cerebral NMR and on liquor analysis was normal.

He also realized an electroencephalogram (EEG) and presented slowing down of the base activity and had practically continued epileptic activity in the temporal and frontal lobes. With the addition of valproic acid (VPA) $750 \mathrm{mg}$ per day the epileptic activity ceased and his status improved one month later. Paliperidone was reintroduced due to residual delusional activity, even though the anti-epileptic was in therapeutic levels. The control EEG showed no epileptic activity but he presented a sequelae encephalopathy trace.

Four months later his admission the patient was discharged, medicated with paliperidone and VPA, and was completely organized, with no psychotic symptomatology, although he had no insight for the episode. 
After 4 months of ambulatory treatment, the patient abandoned his medication and returned to present psychotic symptoms, with persecutory delusions, passivity phenomena and insomnia. He was admitted one more time and, even though the EEG was not performed due to hospital unavailability, the VPA and paliperidone were reintroduced, with remission of his symptoms and discharge after 1 month of treatment.

\section{Discussion}

The differentiation between Psychosis associated with Epilepsy according to the temporal relation with epileptic seizures has clinical and prognostic utility, with Epilepsy-associated psychoses being divided into peri-ictal/acute seizures with a close temporal relationship to epileptic seizures and interictal/chronic seizures, which are characterized by isolated psychotic episodes not related to seizures $[6,11]$.

Peri-ictal psychoses can be divided into pre-ictal psychosis, which occurs before the convulsive episode, ictal, when the psychotic symptoms are the clinical expression of the seizure or as a postictal crisis, occurring up to $120 \mathrm{~h}$ after the seizure episode $[6,11]$.

Interictal psychosis may present as an acute episode that lasts for days or weeks, or as a chronic pathology that lasts for months or years [12].

The apparent clinical differences between Schizophrenia-like Psychosis in neurological diseases and Schizophrenia indeed are quite illusory, especially when Kurt Schneider's firstorder symptoms are used, and transitional states are frequent. Since interictal symptoms are not related to any "convulsive side effects," psychiatric manifestations are very similar to the phenomenological manifestations of Schizophrenia, which is why chronic interictal psychosis is also referred to as Schizophrenia-like Epilepsy Psychosis [5,13].

In contrast to Schizophrenia, the interictal psychotic episode has later onset of symptoms and is characterized by the absence of negative symptoms, a better premorbid condition and improbable personality deterioration, with delusions and hallucinations of being less bizarre and without loss of contact with reality [13-15].

The distinction of Schizophrenia also occurs through the common religious staining of paranoid ideas in patients with Psychotic associated with Epilepsy and, although auditory hallucinations are common, visual hallucinations are relatively rare $[16,17]$.

Frequent symptoms in patients with Psychosis associated with Epilepsy also include psychic or experiential phenomena such as an intellectual aura or dream states, complex visual or auditory hallucinations or illusions, memory flashbacks, déjà vu, jamais vu, and significant emotional states, most commonly, with pronounced fear [18].

Sometimes the patient has insight for the episode and this can also distinguish from Psychotic disorders [19].

Several abnormalities throughout the brain have been described in Temporal Lobe Epilepsy and Schizophrenia, with a number of studies reporting structural abnormalities and genetic mutations presented in both pathologies [4,20-22].

The most accepted theory today is that both Psychosis and Epilepsy are the consequence of underlying neuropathophysiological dysfunction. In some patients this may be Epilepsy, whereas in others, symptomatology is congruent with Schizophrenia and a third group may suffer from both. This theory goes to meet the specter of a "continuum" of Psychosis [23,24].

Aberrant limbic activity and frontal dysfunction play a key role in the neuropathogeny of Psychosis. Variability in the structure and function of frontal lobe areas and adjacent regions (or involvement of these areas by the spread of epileptiform discharges) as well as bitemporal dysfunction may partially explain why some, but not all, patients with Epilepsy develop Psychosis [25].

The treatment of peri-ictal disorders is based on the control of epileptic seizures, whereas the treatment of interictal disorders is more similar to those of purely psychiatric disorders. Strict control of seizures may not only improve the patient's quality of life and dysfunction, but also prevent the development of an interictal psychosis, so it is extremely important to establish a treatment protocol for these cases [6].

NCSE is defined as a seizure-free epileptic persistent condition, lasting more than $30 \mathrm{~min}$, associated with continuous or quasi-continuous epileptiform discharges in the EEG. This condition, because is seizure free, is frequently misdiagnosed [26].

The EEG is necessary to make a definitive diagnosis, but this can be made difficult due 
to the low accessibility to the examination, especially to the scarily monitoring EEG availability, the poor adhesion of the patient and the interpretation of the results, which do not always lead to a definitive diagnosis and are a subject of great controversy. As a consequence, it may be difficult to establish a precise incidence and the published estimates vary widely [27].

The NCSE can have a variety of clinical presentations. It may present as confusion with a floating pattern that is difficult to distinguish from other causes of delirium, and it is possible to have episodes of cognitive impairment interspersed with periods of almost normal functioning, such as a persistent confusional state or as in coma $[28,29]$.

Being similar to other causes of delirium, a cognitive deficit that is particularly likely to affect attention is likely to occur, with individuals easily distracted and having trouble following complex conversations. The duration can be from hours to days [30].

NCSE can be caused by primary epilepsy or seizures secondary to a different pathology, such as cerebrovascular disease, meningitis/ encephalitis, head injury, brain tumor, neurosurgery or cerebral anoxia. The recent longterm discontinuation of benzodiazepines should also increase suspicion $[31,32]$.

A clinical improvement after administration of anti-epileptics, such as an intravenous benzodiazepine, is suggestive of NCSE. Careful monitoring and care availability should be considered in a more specialized unit. Ideally, drugs should be administered during continuous EEG recording to aid diagnostic accuracy. An alternative would be to repeat an EEG after treatment to demonstrate resolution of epileptic activity. There may be clinical situations where timely EEG is not possible and empirical treatment with clinical response monitoring may be warranted. In some cases, a diagnosis may depend only on clinical evaluation [27].

A clinical and/or EEG improvement after treatment makes the diagnosis of NCSE more likely. In some situations, this will be a valuable diagnostic test. In making a diagnosis, specialized interpretation of the tracing is necessary, but ultimately, clinical evaluation, rather than exact criteria, is the diagnostic key [27].

A good outcome may be associated with early and appropriate treatment [33]. Any delay in treatment may decrease the likelihood of a
NCSE reversal and therefore have a detrimental effect on recovery [10].

\section{Conclusion}

In this patient in particular, it is difficult to differentiate the type of Psychosis associated with Epilepsy, since a video-EEG was not performed to specify the duration of the epileptic activity and its relationship with the clinic due to hospital limitations. His presentation can fit into ictal, post-ictal and interictal psychosis.

Regarding the NCSE, the confusional state and the benzodiazepines discontinuation raised that hypothesis, and an abnormal EEG and a clinical response along with correction of EEG abnormalities with VPA clarified the diagnosis, being that the most likely hypothesis TFLE with seizures interspersed with NCSE.

However, there is still the remaining doubt of Schizophrenia co-morbidity, which will only be possible to elucidate with this case progression.

Currently, Psychosis associated with Epilepsy and NCSE are conditions most frequently detected, reflecting the complex relationships between Epilepsy and Psychotic disorders and represents a clinical and therapeutic challenge $[5,8,13]$.

The relatively high prevalence of psychotic symptoms in patients with Epilepsy suggests the existence of pathogenic mechanisms common to both, which may explain the bidirectional relationship and the higher occurrence of comorbidity. However, few studies have addressed this issue and it remains a challenge to characterize neurobiological changes that contribute to the genesis or maintenance of Epilepsy-associated Psychosis, and there is a clear need for further investigation to elucidate local and disseminated dysfunction of neuronal circuits that may be involved $[5,13]$.

Rigorous control of seizures may, in addition to improving patients' quality of life and dysfunction, prevent the development of chronic interictal psychosis [6].

Adequate recognition and treatment of Psychosis in Epilepsy and NCSE are essential for patient management due to the considerable weight on the quality of life and patient's morbidity [10,34].

\section{Conflicts of Interest}

The authors report no conflicts of interest. 


\section{References}

1. Bertram EH. Temporal lobe epilepsy: where do the seizures really begin? Epilepsy. Behav 14(1), 32-37 (2009).

2. Gaitatzis A, Trimble MR, Sander JW. The psychiatric comorbidity of epilepsy. Acta. Neurol. Scand 110(4), 207-220 (2004).

3. Swinkels WAM, Kuyk J, Van Dyck R, et al. Psychiatric comorbidity in epilepsy. Epilepsy. Behav 7(1), 37-50 (2005).

4. Clancy MJ, Clarke MC, Connor DJ, et al. The prevalence of psychosis in epilepsy; a systematic review and meta-analysis. BMC Psychiatry 14(1), 75 (2014).

5. Kanner AM, Rivas-Grajales AM. Psychosis of epilepsy: a multifaceted neuropsychiatric disorder. CNS. Spectr 21(3), 247-257 (2016).

6. Mingot CG, Villar MG, Medel DC, et al. Psicosis epiléptica periictal, una causa de psicosis reversible. Neurología 28(2), 81-87 (2013).

7. DeLorenzo RJ, Waterhouse EJ, Towne AR, et al. Persistent nonconvulsive status epilepticus after the control of convulsive status epilepticus. Epilepsia 39(8), 833-840 (1998).

8. Kaplan PW. Assessing the outcomes in patients with nonconvulsive status epilepticus: nonconvulsive status epilepticus is underdiagnosed, potentially overtreated, and confounded by comorbidity. J. Clin. Neurophysiol 116(4), 341-352 (1999).

9. Granner MA, Ik Lee $\mathrm{S}$. Nonconvulsive status epilepticus: EEG analysis in a large series. Epilepsia 35(1), 42-47 (1994).

10. Kang BS, Jhang Y, Kim Y-S, et al. Etiology and prognosis of non-convulsive status epilepticus. J. Clin. Neurosci 21(11), 1915-1919 (2014).

11. Kanner AM. Psychosis of epilepsy: a neurologist's perspective. Epilepsy. Behav 1(4), 219-227 (2000).

12. Adachi N, Kato $M$, Sekimoto $M$, et al. Recurrent postictal psychosis after remission of interictal psychosis: further evidence of bimodal psychosis. Epilepsia 44(9), 1218-1222 (2003).

13. Kandratavicius L, Hallak JE, Leite JP. What are the similarities and differences between schizophrenia and schizophrenia-like psychosis of epilepsy? A neuropathological approach to the understanding of schizophrenia spectrum and epilepsy. Epilepsy. Behav 38(1), 143-147 (2014).

14. Arzy S, Schurr R. "God has sent me to you": Right temporal epilepsy, left prefrontal psychosis. Epilepsy. Behav 60(1), 7-10 (2016).

15. McCrae N, Whitley R. Exaltation in temporal lobe epilepsy: neuropsychiatric symptom or portal to the divine? J. Med. Humanit 35(3), 241-255 (2014).

16. Slater E, Beard AW. The schizophrenia-like psychoses of epilepsy. Br. J. Psychiatry 109(458), 95-112 (1963).

17. Fujii D, Ahmed I. Psychotic disorder following traumatic brain injury: a conceptual framework. Cognit. Neuropsychiatry 7(1), 41-62 (2002).

18. Penfield W, Perot P. THE BRAIN'S RECORD OF AUDITORY AND VISUAL EXPERIENCE 1: A FINAL SUMMARY AND DISCUSSION. Brain 86(4), 595-696 (1963).

19. Gloor P, Olivier A, Quesney LF, et al. The role of the limbic system in experiential phenomena of temporal lobe epilepsy. Ann. Neurol 12(2), 129-144 (1982).

20. Cascella NG, Schretlen DJ, Sawa A. Schizophrenia and epilepsy: is there a shared susceptibility? Neurosci. Res 63(4), 227-235 (2009).

21. Helbig I, Mefford HC, Sharp AJ, et al. Microdeletions increase risk of idiopathic generalized epilepsy. Nat. Genet 41(2), 160162 (2009).

22. Sundram F, Cannon M, Doherty CP, et al. Neuroanatomical correlates of psychosis in temporal lobe epilepsy: voxel-based morphometry study. Br. J. Psychiatry 197(6), 482-492 (2010).

23. Sachdev P. Schizophrenia-like psychosis and epilepsy: the status of the association. Am. J. Psychiatry 155(3), 325-336 (1998).

24. Binbay T, Drukker M, Elbi H, et al. Testing the psychosis continuum: differential impact of genetic and nongenetic risk factors and comorbid psychopathology across the entire spectrum of psychosis. Schizophr. Bull 38(5), 992-1002 (2011).

25. Hilger E, Zimprich F, Pataraia E, et al. Psychoses in epilepsy: A comparison of postictal and interictal psychoses. Epilepsy. Behav 60, 58-62 (2016).

26. Sutter R, Kaplan PW. Electroencephalographic criteria for nonconvulsive status epilepticus: synopsis and comprehensive survey. Epilepsia 53(s3), 1-51 (2012).

27. Woodford HJ, George J, Jackson M. Non-convulsive status epilepticus: a practical approach to diagnosis in confused older people. Postgrad. Med. J 91(1081), 655-661 (2015).

28. Walker MC, Cockerell OC, Sander J. Non-convulsive status epilepticus presenting as a psychiatric condition. J. R. Soc. Med 89(2), 91-92 (1996).

29. Haffey S, McKernan A, Pang K. Non-convulsive status epilepticus: a profile of patients diagnosed within a tertiary referral centre. $J$. Neurol. Neurosurg. Psychiatry 75(7), 1043-1044 (2004).

30. Drislane FW. Presentation, evaluation, and treatment of nonconvulsive status epilepticus. Epilepsy. Behav 1(5), 301-314 (2000).

31. Thomas $P$, Beaumanoir $A$, Genton $P$, et al. "De novo"absence status of late onset Report of 11 cases. Neurology 42(1), 104-104 (1992).

32. Fernández-Torre JL, Díaz-Castroverde AG. Non-convulsive status epilepticus in elderly individuals: report of four representative cases* Presented in part as a poster at the Fifth Annual Meeting of the Sociedad Asturiana de Neurología, Oviedo, 17 May 2002. Age. Ageing 33(1), 78-81 (2004).

33. Treiman DM, Meyers PD, Walton NY, et al. A comparison of four treatments for generalized convulsive status epilepticus. N. Engl. J. Med 339(12), 792-798 (1998).

34. De Boer HM, Mula M, Sander JW. The global burden and stigma of epilepsy. Epilepsy. Behav 12(4), 540-546 (2008). 